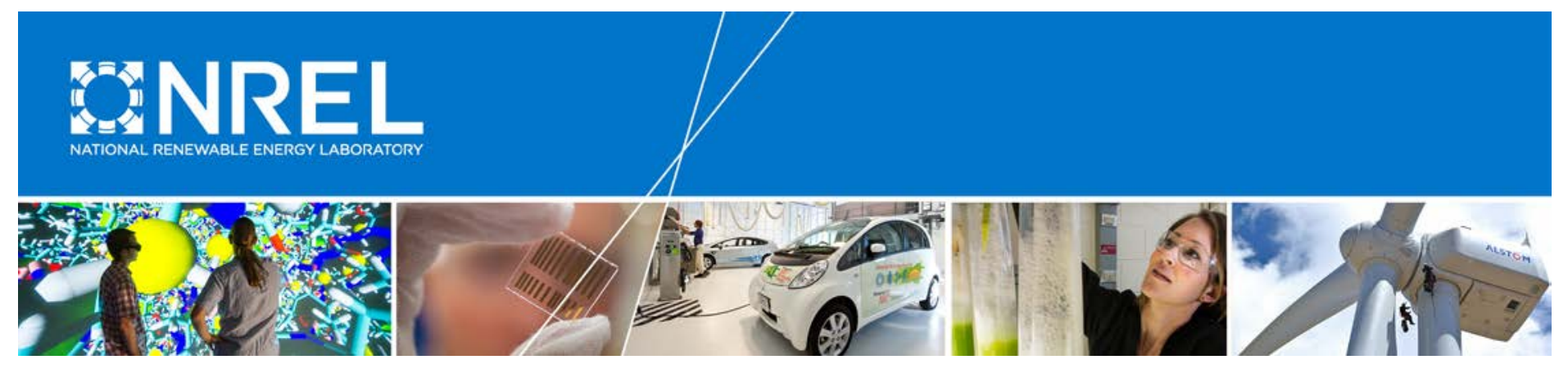

\title{
Incentive-Based Voltage Regulation in Distribution Networks
}

\section{Preprint}

\author{
Xinyang Zhou and Lijun Chen \\ University of Colorado
}

\section{Emiliano Dall'Anese and Kyri Baker National Renewable Energy Laboratory}

To be presented at the 2017 American Control Conference Seattle, Washington

May 24-26, 2017

(C) 2017 IEEE. Personal use of this material is permitted. Permission from IEEE must be obtained for all other uses, in any current or future media, including reprinting/republishing this material for advertising or promotional purposes, creating new collective works, for resale or redistribution to servers or lists, or reuse of any copyrighted component of this work in other works.

NREL is a national laboratory of the U.S. Department of Energy Office of Energy Efficiency \& Renewable Energy Operated by the Alliance for Sustainable Energy, LLC

This report is available at no cost from the National Renewable Energy Laboratory (NREL) at www.nrel.gov/publications.

\section{Conference Paper}

NREL/CP-5D00-67884

March 2017 


\section{NOTICE}

The submitted manuscript has been offered by an employee of the Alliance for Sustainable Energy, LLC (Alliance), a contractor of the US Government under Contract No. DE-AC36-08GO28308. Accordingly, the US Government and Alliance retain a nonexclusive royalty-free license to publish or reproduce the published form of this contribution, or allow others to do so, for US Government purposes.

This report was prepared as an account of work sponsored by an agency of the United States government. Neither the United States government nor any agency thereof, nor any of their employees, makes any warranty, express or implied, or assumes any legal liability or responsibility for the accuracy, completeness, or usefulness of any information, apparatus, product, or process disclosed, or represents that its use would not infringe privately owned rights. Reference herein to any specific commercial product, process, or service by trade name, trademark, manufacturer, or otherwise does not necessarily constitute or imply its endorsement, recommendation, or favoring by the United States government or any agency thereof. The views and opinions of authors expressed herein do not necessarily state or reflect those of the United States government or any agency thereof.

This report is available at no cost from the National Renewable Energy Laboratory (NREL) at www.nrel.gov/publications.

Available electronically at SciTech Connect http:/www.osti.gov/scitech

Available for a processing fee to U.S. Department of Energy and its contractors, in paper, from:

U.S. Department of Energy

Office of Scientific and Technical Information

P.O. Box 62

Oak Ridge, TN 37831-0062

OSTI http://www.osti.gov

Phone: 865.576.8401

Fax: 865.576.5728

Email: reports@osti.gov

Available for sale to the public, in paper, from:

U.S. Department of Commerce

National Technical Information Service

5301 Shawnee Road

Alexandria, VA 22312

NTIS http://www.ntis.gov

Phone: 800.553 .6847 or 703.605 .6000

Fax: 703.605.6900

Email: orders@ntis.gov 


\title{
Incentive-based Voltage Regulation in Distribution Networks
}

\author{
Xinyang Zhou, Emiliano Dall'Anese, Lijun Chen and Kyri Baker
}

\begin{abstract}
This paper considers distribution networks featuring distributed energy resources, and designs an incentivebased algorithm that allows the network operator and the endcustomers to pursue given operational and economic objectives, while concurrently ensuring that voltages are within prescribed limits. This social-welfare maximization problem is challenging due to the non-convexity. We first reformulate the problem as a convex task together with an incentive signal design strategy, and then propose a distributed algorithm for solving the reformulated problem. By doing so, we are able to achieve the solution of the original non-convex problem without exposure of any private information between end-customers and network operator. Stability of the proposed schemes is analytically established and numerically corroborated.
\end{abstract}

Index Terms - Voltage regulation, real-time pricing, social welfare maximization, distribution networks.

\section{INTRODUCTION}

Market-based mechanisms to control distributed energy assets have been recently developed with the objective of incentivizing end-customers to provide ancillary services to the grid while maximizing their own economic benefits; see, e.g., [1]-[4] and the references therein. However, demandresponse and market-based problem formulations do not generally consider the power flows in the distribution network and, hence, they are oblivious to the voltage fluctuations that emerge from the adjustments in the powers generated/consumed. The frameworks proposed in, e.g., [5][7] offer a way to account for the nonlinear power flows, but either their applicability is limited to a restricted class of network topologies, or, they consider only controllability of the (net) real powers.

This paper aims to design an incentive-based algorithm that allows network operator and end-customers to pursue given operational and economic objectives and, in doing so, ensures that voltage magnitudes are within the prescribed limits (e.g., ANSI C84.1 limits). Similar to, e.g., [4], the paper first formulates a social-welfare maximization problem

X. Zhou and L. Chen are with the College of Engineering and Applied Science, University of Colorado, Boulder, CO 80309, USA (Emails: \{Xinyang.Zhou, Lijun.Chen\}@ colorado.edu).

E. Dall'Anese and K. Baker are with the National Renewable Energy Laboratory, Golden, CO 80401, USA (Emails: \{Emiliano.DallAnese, Kyri.Baker\}@nrel.gov)

This work was supported by the U.S. Department of Energy under Contract No. DE-AC36-08GO28308 with the National Renewable Energy Laboratory. Funding provided by the Department of Energy Grid Modernization Laboratory Consortium and by the Laboratory Directed Research and Development Program at the National Renewable Energy Laboratory. The U.S. Government retains and the publisher, by accepting the article for publication, acknowledges that the U.S. Government retains a nonexclusive, paid-up, irrevocable, worldwide license to publish or reproduce the published form of this work, or allow others to do so, for U.S. Government purposes. that captures a variety of optimization objectives, hardware constraints, and the nonlinear power-flow equations governing the physics of distribution systems; for the latter, a linear approximation of the nonlinear power-flow equations [8] [11] is utilized in order to enable the development of a computationally-tractable optimal coordination method. To solve a well-defined but non-convex social-welfare maximization problem, we address two major challenges in this paper, i.e., non-convexity and privacy preservation. 1) We reformulate the non-convex social-welfare maximization problem into a convex problem. Together with an incentive signal design strategy, we are able to achieve the solution of the original non-convex problem by solving the reformulated convex problem. 2) We propose an iterative distributed algorithm where end-customers and network operator achieve consensus on a set of net real and reactive powers that optimize the objectives of both parties while ensuring that voltages are within the prescribed limits. In our design, end-customers are not required to share private information regarding their cost functions and the operating region of their loads/generators with the distribution-network operator.

In the proposed algorithm, the design of the incentive signals is based on the equivalence between the optimal points of the target (non-convex) social-welfare maximization problem and the ones of the subproblems solved at the network operator and the end-customer sides. The development of the iterative distributed algorithm, grounded on the decomposability of the primal-dual projected gradient algorithms, solves the social-welfare maximization problem without exposure of private information from either party to the other. Convergence of the distributed algorithm to the optimizer of a regularized Lagrangian function associated with the social-welfare maximization problem is shown by leveraging contraction mapping arguments. Finally, we characterize the performance of the proposed algorithm in the purview of the nonlinear power-flow equations.

It should be pointed out that, traditionally, voltage regulation problems arising from reverse power flows [12] have been tackled by considering local Volt/VAR and Volt/Watt controllers [13]-[18] or optimization-based techniques [8], [19], [20] that leverage the flexibility of power-electronicsinterfaced renewable sources of energy in adjusting the output real and reactive powers. Compared to local control strategies, the proposed method allows the network operator and the end-customers to pursue well-defined performance objectives; compared to existing optimization strategies, the proposed method casts the voltage regulation problem within an incentive realm, and provides insights as to how to design real-time incentive schemes. 
TABLE I: Notation.

\begin{tabular}{ll}
\hline $\mathcal{N}$ & Set of nodes, excluding node $0, \mathcal{N}:=\{1, \ldots, N\}$ \\
$\mathcal{L}$ & Set of distribution lines \\
$p_{i}, q_{i}$ & Net real and reactive power injected at node $i$ \\
$z_{i}$ & Overall power injected at node $i, z_{i}:=\left[p_{i}, q_{i}\right]^{T}$ \\
$p, q$ & $p:=\left[p_{1}, \ldots, p_{N}\right]^{T}, q:=\left[q_{1}, \ldots, q_{N}\right]^{T}$ \\
$z$ & $z:=\left[p^{T}, q^{T}\right]^{T}$ \\
$V_{i}$ & Complex voltage at node $i$ \\
$v_{i}$ & Voltage magnitude at node $i$ \\
$v$ & $v:=\left[v_{1}, \ldots, v_{N}\right]^{T}$ \\
$\alpha_{i}, \beta_{i}$ & Signal for real and reactive power for node $i$ \\
$s_{i}$ & Overall signal for node $i, z_{i}:=\left[\alpha_{i}, \beta_{i}\right]^{T}$ \\
$\alpha, \beta$ & $\alpha:=\left[\alpha_{1}, \ldots, \alpha_{N}\right]^{T}, \beta:=\left[\beta_{1}, \ldots, \beta_{N}\right]^{T}$ \\
{$[x]_{+}$} & Projection of $x$ onto the non-negative orthant \\
{$[x]_{\mathcal{Z}}$} & Projection of $x$ onto the convex set $\mathcal{Z}$ \\
\hline
\end{tabular}

\section{System Model And Modeling Assumptions}

Consider a distribution network with $N+1$ nodes collected in the set $\mathcal{N} \cup\{0\}$ with $\mathcal{N}:=\{1, \ldots, N\}$ and distribution lines collected in the set $\mathcal{E}$. Let $p_{i} \in \mathbb{R}$ and $q_{i} \in \mathbb{R}$ denote the net real and reactive power injections at node $i \in \mathcal{N}$, $V_{i} \in \mathbb{C}$ the phasor for the line-to-ground voltage at node $i$, and define $v_{i}:=\left|V_{i}\right|$.

Let $\mathcal{Z}_{i}$ denote the feasible set of real and reactive power $p_{i}$ and $q_{i}$ at node $i \in \mathcal{N}$. Since $p_{i}$ and $q_{i}$ are aggregate net powers at node $i$, the set $\mathcal{Z}_{i}$ is assumed convex and compact for all $i \in \mathcal{N}$. This is the case for, e.g., photovoltaic (PV) systems [8], energy storage systems, small-scale diesel generators, and variable frequency drives; for DERs with discrete levels of output powers, $\mathcal{Z}_{i}$ represents the convex envelope of the possible operating points; see, e.g., [21].

Voltages, currents, and powers $\left\{p_{i}, q_{i}\right\}$ are related by the well-known nonlinear AC power flow equations; assuming, for illustrative purpose, a balanced tree network, these equations read:

$$
\begin{aligned}
P_{i j} & =-p_{j}+\sum_{k:(j, k) \in \mathcal{L}} P_{j k}+r_{i j} \ell_{i j} \\
Q_{i j} & =-q_{j}+\sum_{k:(j, k) \in \mathcal{L}} Q_{j k}+x_{i j} \ell_{i j} \\
v_{j}^{2} & =v_{i}^{2}-2\left(r_{i j} P_{i j}+x_{i j} Q_{i j}\right)+\left(r_{i j}^{2}+x_{i j}^{2}\right) \ell_{i j} \\
\ell_{i j} v_{i}^{2} & =P_{i j}^{2}+Q_{i j}^{2}
\end{aligned}
$$

where $\ell_{i j}$ is the squared magnitude of the current on line $(i, j), P_{i j}, Q_{i j}$ are real and reactive powers injected on line $(i, j)$, and $r_{i j}+\mathrm{j} x_{i j}$ is the admittance on line $(i, j)$. However, a linear approximation of the nonlinear power-flow equations is utilized in order to develop a computationallytractable voltage-control algorithm. Particularly, the following linearized relationship between voltage magnitudes and injected powers is utilized:

$$
\hat{v} \approx R p+X q+a,
$$

where the positive definite resistance and reactance matrices $R, X \in \mathbb{R}_{++}^{N \times N}$ and the vector $a \in \mathbb{R}^{N}$ are system parameters that can be formed as described in, e.g., [8], [9], [11], [22].

Remark 1. For simplicity of exposition, the framework is outlined for a single-phase system; however, the proposed method can be readily extended to multi-phase settings. This can be obtained by utilizing, for example, a multi-phase extension of (2) of a semidefinite relaxation approach for multi-phase systems [23], [24].

\section{A. Problem setup}

The objective is to design a strategy where the network operator and the end-customers pursue their own operational and economic objectives, while achieving a global coordination to enforce voltage regulation.

1) End-customers' problem: Consider a cost function $C_{i}\left(p_{i}, q_{i}\right)$ that captures well-defined performance objectives for the customer(s) located at node $i \in \mathcal{N}$. Let $\alpha_{i} \in \mathbb{R}$ and $\beta_{i} \in \mathbb{R}$ be the incentive signals sent by the network operator (e.g., distribution system operator or aggregator) for real and reactive power injections, respectively. Given signals $\left(\alpha_{i}, \beta_{i}\right)$, the following optimization problem is solved at each node $i \in \mathcal{N}$ :

$$
\begin{array}{rll}
\left(\mathcal{P}_{1, i}\right) & \min _{p_{i}, q_{i}} & f_{i}\left(p_{i}, q_{i} \mid \alpha_{i}, \beta_{i}\right), \\
\text { s.t. } & \left(p_{i}, q_{i}\right) \in \mathcal{Z}_{i},
\end{array}
$$

where $f_{i}\left(p_{i}, q_{i} \mid \alpha_{i}, \beta_{i}\right):=C_{i}\left(p_{i}, q_{i}\right)-\alpha_{i} p_{i}-\beta_{i} q_{i}$ with $\alpha_{i} p_{i}$ and $\beta_{i} q_{i}$ representing payment to/reward from the network operator. The following assumption is made.

Assumption 1 Functions $C_{i}\left(p_{i}, q_{i}\right), \forall i \in \mathcal{N}$ are continuously differentiable and strongly convex in $\left(p_{i}, q_{i}\right)$. Moreover, the first-order derivative of $C_{i}\left(p_{i}, q_{i}\right)$ is bounded in $\mathcal{Z}_{i}$.

The assumption of bounded derivative means that an infinitesimal change should not lead to a jump in cost. Since (3a) is strongly convex in both $p_{i}$ and $q_{i}$ and $\mathcal{Z}_{i}$ is convex and compact, a unique solution $\left(p_{i}^{*}, q_{i}^{*}\right)$ exists. For future developments, consider a so-called best-response strategy $b_{i}$ of end-customer $i$ as the following function of $\alpha_{i}$ and $\beta_{i}$ :

$$
\left(p_{i}^{*}, q_{i}^{*}\right)=b_{i}\left(\alpha_{i}, \beta_{i}\right):=\arg \min _{\left(p_{i}, q_{i}\right) \in \mathcal{Z}_{i}} f_{i}\left(p_{i}, q_{i} \mid \alpha_{i}, \beta_{i}\right) .
$$

Notice further that, for illustrative simplicity, problem $\left(\mathcal{P}_{1, i}\right)$ is stated in terms of net powers; it is clear that if multiple controllable devices are located at node $i$, problem $\left(\mathcal{P}_{1, i}\right)$ should include optimization variables capturing the power consumption/injection of each device.

2) Social-welfare problem: Consider a cost function $D(\hat{v})$ capturing the network-oriented objective in voltage. For instance, to minimize the voltage deviation from the nominal value $v^{\text {nom }}$, we can set $D(\hat{v})=\frac{1}{2}\left\|\hat{v}-v^{\text {nom }}\right\|^{2}$. Let $\mathcal{Z}:=$ $\mathcal{Z}_{1} \times \ldots \times \mathcal{Z}_{N}$, and the following assumption is made.

Assumption 2 Function $D(\hat{v})$ is continuously differentiable, convex, and with bounded first-order derivative in $\mathcal{Z}$.

Consider the following social-welfare optimization problem that captures both customer-oriented and network- 
oriented objectives of a (portion of a) distribution feeder:

$$
\begin{aligned}
\left(\mathcal{P}_{2}\right) \min _{p, q, \hat{v}, \alpha, \beta} & \sum_{i \in \mathcal{N}} C_{i}\left(p_{i}, q_{i}\right)+\gamma D(\hat{v}), \\
\text { s.t. } \quad & \hat{v}=R p+X q+a, \\
& \underline{v} \leq \hat{v} \leq \bar{v}, \\
& \left(p_{i}, q_{i}\right)=b_{i}\left(\alpha_{i}, \beta_{i}\right), \forall i \in \mathcal{N},
\end{aligned}
$$

where $\gamma \in \mathbb{R}_{+}$is a parameter used to trade off between the end-customers' and the network-oriented objectives, while vectors $\underline{v}$ and $\bar{v}$ are prescribed minimum and maximum voltage magnitude limits (e.g., ANSI C84.1 limits). Notice that the total payment from/reward to the end-customers cancels out the payment received/reward paid by the network operator, and is therefore not explicitly shown in the social welfare objective function (5a).

Also notice that the best-response strategy of $\left(\mathcal{P}_{1, i}\right)$ is embedded in $\left(\mathcal{P}_{2}\right)$ through the constraint $(5 \mathrm{~d})$, i.e., the network operator knows the reaction of the end-customers towards any incentive and takes it into consideration when making decisions. This constitutes a Stackelberg game where the network operator solves $\left(\mathcal{P}_{\mathbf{2}}\right)$ and sends the signals $\alpha$ and $\beta$ to all end-customers $i \in \mathcal{N}$; subsequently, each endcustomer responds with computed power injections $\left(p_{i}^{*}, q_{i}^{*}\right)$ from $\left(\mathcal{P}_{1, i}\right)$ based on the received signals $\left(\alpha_{i}, \beta_{i}\right)$. By design, $\left(p^{*}, q^{*}\right)$ coincides with the optimal solution of $\left(\mathcal{P}_{\mathbf{2}}\right)$.

However, it is impractical to solve problem $\left(\mathcal{P}_{2}\right)$ not only because of its non-convexity introduced by constraints (5d), but also because of its requirement for end-customers' full information, which is usually private. In the following section, we first reformulate $\left(\mathcal{P}_{\mathbf{2}}\right)$ as a convex optimization problem with a signal design strategy; they together bypass the problem of non-convexity, while achieving the solution of problem $\left(\mathcal{P}_{2}\right)$. Then based on primal-dual gradient algorithm, we propose a distributed algorithm that prevents any exposure of private information from the end-customers while solving a regularized reformulated convex optimization problem.

\section{INCENTIVE-BASED VOLTAGE REGULATION}

\section{A. Convex reformulation and incentive design}

In the following, a one-step mechanism will be designed, where the end-customers submit to the network operator a "bid" in the form of their cost function $C_{i}(\cdot)$ as well as their operating region $\mathcal{Z}_{i}$; upon receiving the incentive signals from the network operator, the end-customers compute the real and reactive powers via $\left(\mathcal{P}_{1, i}\right)$.

To this end, consider the following convex optimization problem:

$$
\begin{aligned}
\left(\mathcal{P}_{\mathbf{3}}\right) \min _{p, q, \hat{v}} & \sum_{i \in \mathcal{N}} C_{i}\left(p_{i}, q_{i}\right)+\gamma D(\hat{v}), \\
\text { s.t. } \quad & \hat{v}=R p+X q+a, \\
& \underline{v} \leq \hat{v} \leq \bar{v}, \\
& \left(p_{i}, q_{i}\right) \in \mathcal{Z}_{i} \quad \forall i \in \mathcal{N},
\end{aligned}
$$

where the non-convex constraint $(5 \mathrm{~d})$ in $\left(\mathcal{P}_{2}\right)$ is replaced with (6d). We assume $\left(\mathcal{P}_{\mathbf{3}}\right)$ is feasible.
Assumption 3 (Slater's condition) There exists a feasible point $(\tilde{p}, \tilde{q}) \in \mathcal{Z}$, such that

$$
\underline{v} \leq R \tilde{p}+X \tilde{q}+a \leq \bar{v} .
$$

Assumption 3 does not involve strict inequality because the constraint is linear. Given the strong convexity of objective function (6a) in $\left(p_{i}, q_{i}\right)$ and the linear relation (6b), a unique optimal solution exists for problem $\left(\mathcal{P}_{\mathbf{3}}\right)$. Notice that a solution $\left(p_{i}^{*}, q_{i}^{*}, \hat{v}^{*}\right)$ of $\left(\mathcal{P}_{\mathbf{3}}\right)$ may not be feasible for $\left(\mathcal{P}_{\mathbf{2}}\right)$, i.e., there does not exist a $\left(\alpha^{*}, \beta^{*}\right)$ such that $\left(p_{i}^{*}, q_{i}^{*}\right)=b_{i}\left(\alpha_{i}^{*}, \beta_{i}^{*}\right)$. We will, however, show next that such $\left(\alpha^{*}, \beta^{*}\right)$ exists, and thus the solution of $\left(\mathcal{P}_{\mathbf{3}}\right)$ gives the solution of $\left(\mathcal{P}_{2}\right)$.

Substitute (6b) into (6c), and denote by $\mu$ and $\bar{\mu}$ the dual variables associated with the constraints $(6 \bar{c})$. Let $\hat{v}^{*}$ be the optimal voltage magnitudes produced by $\left(\mathcal{P}_{\mathbf{3}}\right)$ and denote the optimal dual variables associated with $(6 \mathrm{c})$ as $\mu^{*}, \bar{\mu}^{*}$. Then, the incentive signals are designed as:

$$
\begin{aligned}
\alpha^{*} & =R\left[-\gamma \nabla_{\hat{v}} D\left(\hat{v}^{*}\right)+\left(\underline{\mu}^{*}-\bar{\mu}^{*}\right)\right], \\
\beta^{*} & =X\left[-\gamma \nabla_{\hat{v}} D\left(\hat{v}^{*}\right)+\left(\underline{\mu}^{*}-\bar{\mu}^{*}\right)\right],
\end{aligned}
$$

where $\nabla_{\hat{v}} D$ denotes the gradient of function $D$ with respect to the vector $\hat{v}$. As will be shown next, the above incentive signals are bounded, precluding the possibility of infinitely large signals.

Theorem 1 Under Assumptions 1-3, the incentive signals $\left(\alpha^{*}, \beta^{*}\right)$ defined by equation (7) are bounded.

Proof: Notice that the derivative $\nabla_{\hat{v}} D$ is bounded. To show the boundedness of $\left(\alpha^{*}, \beta^{*}\right)$, it is enough to show that the optimal duals $\left(\mu^{*}, \bar{\mu}^{*}\right)$ are bounded.

Consider the KKT conditions for problem $\left(\mathcal{P}_{\mathbf{3}}\right)$ :

$$
\begin{aligned}
& \left(\nabla_{p} \sum_{i \in \mathcal{N}} C_{i}\left(p_{i}^{*}, q_{i}^{*}\right)+\gamma R \nabla_{\hat{v}} D\left(\hat{v}^{*}\right)-R\left(\underline{\mu}^{*}-\bar{\mu}^{*}\right)\right)^{T} \\
& \cdot\left(p-p^{*}\right) \geq 0, \forall(p, q) \in \mathcal{Z}, \quad \text { (8a) } \\
& \left(\nabla_{q} \sum_{i \in \mathcal{N}} C_{i}\left(p_{i}^{*}, q_{i}^{*}\right)+\gamma X \nabla_{\hat{v}} D\left(\hat{v}^{*}\right)-X\left(\underline{\mu}^{*}-\bar{\mu}^{*}\right)\right)^{T} \\
& \cdot\left(q-q^{*}\right) \geq 0, \forall(p, q) \in \mathcal{Z}, \\
& \hat{v}^{*}=R p^{*}+X q^{*}+a, \\
& \underline{v} \leq \hat{v}^{*} \leq \bar{v} \text {, } \\
& \left(\underline{v}-\hat{v}^{*}\right)^{T} \underline{\mu}^{*}=0, \underline{\mu}^{*} \geq 0, \\
& \left(\hat{v}^{*}-\bar{v}\right)^{T} \bar{\mu}^{*}=0, \bar{\mu}^{*} \geq 0 .
\end{aligned}
$$

Combine (8a)-(8c), and we have

$$
\begin{aligned}
& \left(\nabla_{p} \sum_{i \in \mathcal{N}} C_{i}\left(p_{i}^{*}, q_{i}^{*}\right)+\gamma R \nabla_{\hat{v}} D\left(\hat{v}^{*}\right)\right)^{T}\left(p-p^{*}\right) \\
& +\left(\nabla_{q} \sum_{i \in \mathcal{N}} C_{i}\left(p_{i}^{*}, q_{i}^{*}\right)+\gamma X \nabla_{\hat{v}} D\left(\hat{v}^{*}\right)\right)^{T}\left(q-q^{*}\right) \\
& +\left(\bar{\mu}^{*}-\underline{\mu}^{*}\right)^{T}\left(\hat{v}-\hat{v}^{*}\right) \geq 0, \forall(p, q) \in \mathcal{Z}, \forall \hat{v},
\end{aligned}
$$

where the first two terms on the left of the inequality are bounded because of the bounded derivative of cost functions and the bounded set $\mathcal{Z}$. By the complementary slackness 
condition (8e)-(8f), $\bar{\mu}_{i}^{*}$ and $\underline{\mu}_{i}^{*}, i \in \mathcal{N}$ cannot be nonzero at the same time. If $\bar{\mu}_{i}^{*} \rightarrow \infty$, then $\hat{v}_{i}^{*}=\bar{v}_{i}$ and we can choose a $(p, q)$ and thus $\hat{v}_{i}$ such that the third term on the left of (9) goes to $-\infty$ and (9) does not hold. So, $\bar{\mu}_{i}^{*}$ and thus $\bar{\mu}^{*}$ is bounded. Similarly, we can show that $\mu^{*}$ is bounded too. The result follows.

By examining the optimality conditions of $\left(\mathcal{P}_{\mathbf{2}}\right)$ and $\left(\mathcal{P}_{3}\right)$, we have the following result.

Theorem 2 The solution of problem $\left(\mathcal{P}_{\mathbf{3}}\right)$ along with the signals $\left(\alpha^{*}, \beta^{*}\right)$ defined in equation (7) solves problem $\left(\mathcal{P}_{\mathbf{2}}\right)$.

Proof: By the signal design (7), equations (8a)-(8b) become

$$
\begin{aligned}
& \left(\nabla_{p} \sum_{i \in \mathcal{N}} C_{i}\left(p_{i}^{*}, q_{i}^{*}\right)-\alpha^{*}\right)^{T}\left(p-p^{*}\right) \geq 0 \\
& \left(\nabla_{q} \sum_{i \in \mathcal{N}} C_{i}\left(p_{i}^{*}, q_{i}^{*}\right)-\beta^{*}\right)^{T}\left(q-q^{*}\right) \geq 0, \forall(p, q) \in \mathcal{Z}
\end{aligned}
$$

Notice that the above variational inequalities imply that $\left(p_{i}^{*}, q_{i}^{*}\right)=b_{i}\left(\alpha_{i}^{*}, \beta_{i}^{*}\right), i \in \mathcal{N}$. So, the solution of problem $\left(\mathcal{P}_{3}\right)$ along with $\left(\alpha^{*}, \beta^{*}\right)$ defined in (7) is feasible for problem $\left(\mathcal{P}_{2}\right)$. The result follows, as $\left(\mathcal{P}_{\mathbf{3}}\right)$ is a relaxation of $\left(\mathcal{P}_{2}\right)$.

From now on, we will use the optima of $\left(\mathcal{P}_{\mathbf{3}}\right)$ and $\left(\mathcal{P}_{2}\right)$ interchangeably depending on the context. Next, based on Theorem 2, we will develop an iterative algorithm that achieves the optimum of $\left(\mathcal{P}_{\mathbf{3}}\right)$ (and hence that of $\left(\mathcal{P}_{2}\right)$ ) without exposing any private information of the endcustomers to the network operator.

Remark 3. Theorem 2 asserts that the non-convex problem $\left(\mathcal{P}_{\mathbf{2}}\right)$ can be solved through solving a convex problem $\left(\mathcal{P}_{\mathbf{3}}\right)$. At the first glance, it appears that the non-convexity of $\left(\mathcal{P}_{\mathbf{2}}\right)$ comes from a non-convex representation of the feasible set that may have a convex representation as implied by $\left(\mathcal{P}_{\mathbf{3}}\right)$. Ongoing investigations will identify the specific problem structure to broaden the result in Theorem 2 to a larger class of problems.

\section{B. Design of iterative algorithm}

For notational simplicity, let $s_{i}=\left[\alpha_{i}, \beta_{i}\right]^{T}$ denote the overall signals for end-customer $i$ and define $z_{i}=\left[p_{i}, q_{i}\right]^{T}$. Further denote by $z:=\left[p^{T}, q^{T}\right]^{T} \in \mathbb{R}^{2 N}$ the vector of stacked power injections, and by $\mu:=\left[\underline{\mu}^{T}, \bar{\mu}^{T}\right]^{T} \in \mathbb{R}^{2 N}$ the vector of stacked dual variables. Consider the following Lagrangian function associated with $\left(\mathcal{P}_{\mathbf{3}}\right)$ :

$$
\begin{aligned}
\mathcal{L}(z, \mu)=\sum_{i \in \mathcal{N}} C_{i}\left(z_{i}\right) & +\gamma D(z)+\underline{\mu}^{T}(\underline{v}-R p-X q-a) \\
& +\bar{\mu}^{T}(R p+X q+a-\bar{v})
\end{aligned}
$$

which is obtained by keeping the constraints $z \in \mathcal{Z}$ and $\mu \in \mathbb{R}_{+}^{2 N}$ implicit. To facilitate the development of provably convergent algorithms, consider the following regularized
Lagrangian function:

$$
\begin{aligned}
\mathcal{L}_{r}(z, \mu):= & \sum_{i \in \mathcal{N}} C_{i}\left(z_{i}\right)+\gamma D(z)+\underline{\mu}^{T}(\underline{v}-R p-X q-a) \\
& +\bar{\mu}^{T}(R p+X q+a-\bar{v})-\frac{r}{2}\|\mu\|^{2}
\end{aligned}
$$

where $r>0$ is a predefined parameter (see e.g., [25], [26]). With the regularization term $-\frac{r}{2}\|\mu\|^{2}$, the resultant function $\mathcal{L}_{r}(z, \mu)$ is strongly concave in the dual variables.

Based on (12), consider the following minimax problem:

$$
\max _{\mu \in \mathbb{R}_{+}^{2 N}} \min _{z \in \mathcal{Z}} \mathcal{L}_{r}(z, \mu) .
$$

In general, the unique optimizer of (13) is not a saddle-point of the Lagrangian function (12) because of the regularization term $-\frac{r}{2}\|\mu\|^{2}$; the discrepancy between the unique optimizer of (13) and the optimizers of (12) can be bounded as shown in [25]. However, the key advantage is that primaldual gradient methods applied to (13) exhibit improved convergence properties as explained next.

Consider then the following primal-dual projected gradient method, where $k$ denotes the iteration index:

$$
\begin{aligned}
{\left[\begin{array}{l}
z(k+1) \\
\mu(k+1)
\end{array}\right] } & =\hat{T}\left(\left[\begin{array}{c}
z(k) \\
\mu(k)
\end{array}\right]\right) \\
& =\left[\left[\begin{array}{c}
z(k) \\
\mu(k)
\end{array}\right]-\left[\begin{array}{c}
\varepsilon_{1} \nabla_{z} \mathcal{L}_{r}(z(k), \mu(k)) \\
-\varepsilon_{2} \nabla_{\mu} \mathcal{L}_{r}(z(k), \mu(k))
\end{array}\right]\right]_{\mathcal{Z} \times \mathbb{R}_{+}^{2 N}},
\end{aligned}
$$

where $[x]_{\mathcal{Z} \times \mathbb{R}_{+}^{2 N}}$ denotes the projection of the vector $x$ onto the set $\mathcal{Z} \times \mathbb{R}_{+}^{2 N}$, and $\varepsilon_{1}, \varepsilon_{2}>0$ are prescribed stepsizes for the primal and the dual updates. Notice that $\nabla_{z} \mathcal{L}_{r}(z, \mu)$ and $\nabla_{\mu} \mathcal{L}_{r}(z, \mu)$ are Lipschitz continuous and strongly monotone. Therefore by virtue of [27, Sec. 3.5, Proposition 5.4], the following result holds.

Theorem 3 There exist some $\bar{\varepsilon}_{1}, \bar{\varepsilon}_{2}>0$ such that for any $\varepsilon_{1} \in\left(0, \bar{\varepsilon}_{1}\right], \varepsilon_{2} \in\left(0, \bar{\varepsilon}_{2}\right], \hat{T}$ is a contraction mapping. For $\varepsilon_{1} \in\left(0, \bar{\varepsilon}_{1}\right], \varepsilon_{2} \in\left(0, \bar{\varepsilon}_{2}\right]$, the sequence $\{(z(k), \mu(k))\}$ generated by (14) converges geometrically to the optimizer of (13).

The decomposable structure of (14) naturally enables the following iterative distributed algorithm:

$$
\begin{aligned}
z_{i}(k+1) & =\left[z_{i}(k)-\varepsilon_{1}\left(\nabla C_{i}\left(z_{i}(k)\right)-s_{i}(k)\right)\right]_{\mathcal{Z}_{i}} \\
\underline{\mu}(k+1) & =\left[\underline{\mu}(k)+\varepsilon_{2}(\underline{v}-\hat{v}(k)-r \underline{\mu}(k))\right]_{+} \\
\bar{\mu}(k+1) & =\left[\bar{\mu}(k)+\varepsilon_{2}(\hat{v}(k)-\bar{v}-r \bar{\mu}(k))\right]_{+} \\
\alpha(k+1) & =R\left[\underline{\mu}(k+1)-\bar{\mu}(k+1)-\gamma \nabla_{\hat{v}} D(\hat{v}(k))\right] \\
\beta(k+1) & =X\left[\mu(k+1)-\bar{\mu}(k+1)-\gamma \nabla_{\hat{v}} D(\hat{v}(k))\right], \\
\hat{v}(k+1) & =R p(k+1)+X q(k+1)+a
\end{aligned}
$$

where the power setpoints of each device are computed locally through (15a) and (15b)-(15f) are performed at the network operator. The resultant scheme is tabulated as Algorithm 1. Notice that each end-customer $i$ does not share its cost function $C_{i}$ or its feasible set $\mathcal{Z}_{i}$ with the network operator; rather, the end-customer transmits to the network 


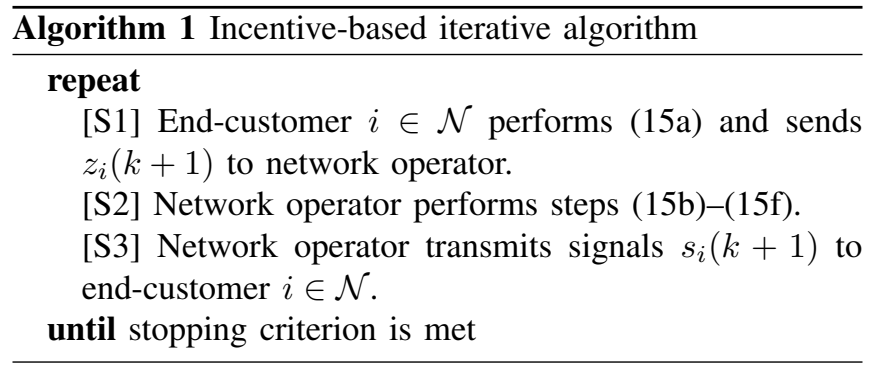

operator only the resultant power injections $z_{i}(k)$. Indeed, the results of Theorem 3 apply to (15) too.

\section{Accounting for nonlinear power flows}

The iterative algorithm (15) was designed and analyzed based on an approximate linear model for the voltages. In this section, the stability of Algorithm 1 is assessed in the purview of the nonlinear AC power-flow model [cf. (1)]. Particularly, the step (15f) is no longer executed to estimate the voltages; rather, the voltages are computed based on a nonlinear AC power-flow model or directly measured from the network.

Hereafter, hatted symbols (e.g., $\hat{v}$ ) refer to variables used in the linearized voltage model; on the other hand, non-hatted symbols represent electrical quantities obeying the nonlinear AC power-flow model. Accordingly, the control strategy is modified as follows:

$$
\begin{aligned}
& z_{i}(k+1)=\left[z_{i}(k)-\varepsilon_{1}\left(\nabla C_{i}\left(z_{i}(k)\right)-s_{i}(k)\right)\right]_{\mathcal{Z}_{i}}, \\
& \underline{\mu}(k+1)=\left[\underline{\mu}(k)+\varepsilon_{2}(\underline{v}-v(k)-r \underline{\mu}(k))\right]_{+}, \\
& \bar{\mu}(k+1)=\left[\bar{\mu}(k)+\varepsilon_{2}(v(k)-\bar{v}-r \bar{\mu}(k))\right]_{+}, \\
& \alpha(k+1)=R\left[\underline{\mu}(k+1)-\bar{\mu}(k+1)-\gamma \nabla_{v} D(v(k))\right], \\
& \beta(k+1)=X\left[\underline{\mu}(k+1)-\bar{\mu}(k+1)-\gamma \nabla_{v} D(v(k))\right] \\
& v(k+1) \quad \text { obey the nonlinear model }(1) .
\end{aligned}
$$

The power setpoints are updated at each node $i \in \mathcal{N}$ via (16a) and commanded to the devices; steps (16b)-(16e) are performed by the the network operator; and, voltages are either computed based on a nonlinear AC power-flow model (e.g., using OpenDSS) or directly measured.

To establish convergence of (16), the following is assumed (see also [28], [29]).

Assumption 4 There exists a constant $e>0$ such that $\left|\hat{v}_{i}(z)-v_{i}(z)\right| \leq e, i \in \mathcal{N}$, for all $z \in \mathcal{Z}$.

This assumption bounds the discrepancy between voltages generated based on the linearized model and the actual voltages (obtained from the nonlinear $\mathrm{AC}$ power-flow equations or measured). By comparing (15) with (16), Assumption 4 naturally leads to the following bounds:

$$
\begin{aligned}
\left|\hat{\mu}_{i}-\underline{\mu}_{i}\right| & \leq \varepsilon_{2} e, \quad\left|\hat{\bar{\mu}}_{i}-\bar{\mu}_{i}\right| \leq \varepsilon_{2} e, \\
\left|\hat{\alpha}_{i}-\alpha_{i}\right| & \leq R_{i}^{T}\left(\gamma \nabla^{2} D(\tilde{v}) \mathbf{1}_{n}+\varepsilon_{2}\right) e, \\
\left|\hat{\beta}_{i}-\beta_{i}\right| & \leq X_{i}^{T}\left(\gamma \nabla^{2} D(\tilde{v}) \mathbf{1}_{n}+\varepsilon_{2}\right) e,
\end{aligned}
$$

for some $\tilde{v}$. The following bounds can be readily shown too:

$$
\begin{aligned}
\left|\hat{p}_{i}-p_{i}\right| & \leq \varepsilon_{1} R_{i}^{T}\left(\gamma \nabla^{2} D(\tilde{v}) \mathbf{1}_{n}+\varepsilon_{2}\right) e:=\delta_{1, i}, \\
\left|\hat{q}_{i}-q_{i}\right| & \leq \varepsilon_{1} X_{i}^{T}\left(\gamma \nabla^{2} D(\tilde{v}) \mathbf{1}_{n}+\varepsilon_{2}\right) e:=\delta_{2, i} .
\end{aligned}
$$

Let $\delta:=\left[\delta_{1,1}, \ldots, \delta_{1, N}, \delta_{2,1}, \ldots, \delta_{2, N}\right] \in \mathbb{R}_{+}^{2 N \times 1}$, and collect the primal and dual variables the vector $y:=(z, \mu)$ for notational simplicity. Consequently, one has that:

$$
\|\hat{T}(y)-T(y)\| \leq\|\rho\|, \forall y \in \mathcal{Z} \times \mathbb{R}_{+}^{2 N},
$$

where $\rho:=\left[\varepsilon_{2} e \cdot \mathbf{1}_{1 \times 2 N}, \delta^{T}\right]^{T}$ and $T(\cdot)$ is the counterpart of $\hat{T}(\cdot)$ for the iterates (16). Let $\Delta \leq \bar{\Delta}<1$ be the contraction modulus for $\hat{T}(\cdot)$; by definition, we have that:

$$
\left\|\hat{T}(y)-\hat{T}\left(y^{\prime}\right)\right\| \leq \Delta\left\|y-y^{\prime}\right\|, \forall y, y^{\prime} \in \mathcal{Z} \times \mathbb{R}_{+}^{2 N},
$$

and the following result can be established.

Theorem 4 Iterates (16) converge to the unique optimizer of (13) within a ball of radius $\frac{\|\rho\|}{1-\Delta}$.

Proof: Let $\hat{y}^{*}$ be the unique optimizer of (13) and consider bounding $\left\|y(k)-\hat{y}^{*}\right\|$ as follows:

$$
\begin{aligned}
& \left\|y(k)-\hat{y}^{*}\right\|=\left\|T(y(k-1))-\hat{y}^{*}\right\| \\
& =\left\|T(y(k-1))-\hat{T}(y(k-1))+\hat{T}(y(k-1))-\hat{y}^{*}\right\| \\
& \leq\|T(y(k-1))-\hat{T}(y(k-1))\|+\left\|\hat{T}(y(k-1))-\hat{y}^{*}\right\| \\
& \leq\|\rho\|+\left\|\hat{T}(y(k-1))-\hat{T}\left(\hat{y}^{*}\right)\right\| \\
& \leq\|\rho\|+\Delta\left\|y(k-1)-\hat{y}^{*}\right\|,
\end{aligned}
$$

where the first inequality is due to the triangle inequality; the second inequality is due to Assumption 4; and the last inequality leverages the definition of contraction mapping. By repeating steps (19)-(20) recursively, one can obtain:

$$
\left\|y(k)-\hat{y}^{*}\right\| \leq \frac{\|\rho\|\left(1-\Delta^{k}\right)}{1-\Delta}+\Delta^{k}\left\|y(0)-\hat{y}^{*}\right\| .
$$

When $k \rightarrow \infty$, one has that:

$$
\lim _{k \rightarrow \infty} \sup \left\|y(k)-\hat{y}^{*}\right\|=\frac{\|\rho\|}{1-\Delta} .
$$

\section{Application Scenarios}

Consider a modified version of the IEEE 37-node test feeder shown in Figure 1. The modified network is obtained by considering a single-phase equivalent, and by replacing the loads specified in the original dataset with real load data measured from feeders in Anatolia, CA during the week of August 2012 [30]. Line impedances, shunt admittances, as well as active and reactive loads are adopted from the respective dataset. It is assumed that eighteen photovoltaic (PV) systems are located at nodes 4, 7, 10, 13, 17, 20, 22, 23, $26,28,29,30,31,32,33,34,35$, and 36 , and their generation profile is simulated based on the real solar irradiance data available in [30]. The rating of these inverters are $300 \mathrm{kVA}$ for $i=3,350 \mathrm{kVA}$ for $i=15,16$, and $200 \mathrm{kVA}$ for the remaining inverters. With this setup, when no actions are taken to prevent over-voltages, one would obtain the voltage snapshot at noon illustrated in Figure 3 (blue dots). 


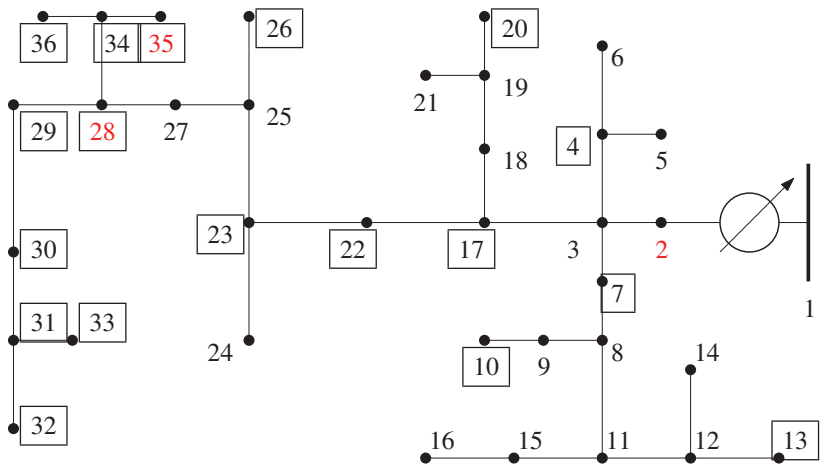

Fig. 1: IEEE 37-node feeder. The boxed nodes represent the location of PV systems. The red nodes are the ones analyzed in the numerical example.

It will be demonstrated how the proposed distributed voltage regulation algorithm can reliably prevent over-voltages that are likely to be experienced during periods when PV generation exceeds the demand [12]. The voltage limits $\bar{v}_{i}$ and $\underline{v}_{i}$ are set to 1.05 p.u. and 0.95 p.u. respectively, for $\forall i \in \mathcal{N}$. Stepsizes $\varepsilon_{1}$ and $\varepsilon_{2}$ are tuned in Section IV-A for the purpose of finding feasible choices, and depicting convergence speed and non-convergence situation as well. The customers' objective functions are set uniformly as $C_{i}\left(p_{i}, q_{i}\right)=c_{p}\left(p_{i, \mathrm{pv}}-p_{i}\right)^{2}+c_{q} q_{i}^{2}$, in an effort to minimize the amount of real power curtailed and the amount of reactive power injected or absorbed. The coefficients are set to $c_{p}=3$ and $c_{q}=1$. The network-oriented objective is set to be $D(v)=\frac{1}{2}\left\|v-v^{\text {nom }}\right\|_{2}^{2}$, penalizing voltage deviation from the nominal value. Without loss of generality, we demonstrate our result with trade-off parameter $\gamma$ set to be 0 or 1 , where the former setup is indifferent about voltage deviation cost $D(v)$ as long as voltages are within acceptable limits, and the latter characterizes an even tradeoff between end-customers' and network-oriented objectives. The regularization parameter $r$ is set to be $10^{-4}$. All the simulations are run with nonlinear $\mathrm{AC}$ power flow model calculated by MATPOWER.

\section{A. Convergence}

In this part, we show the convergence of the iterative voltage regulation algorithm with $\gamma=0$. Similar results with $\gamma=1$ can be observed. By Theorem 3, stepsizes $\varepsilon_{1}$ and $\varepsilon_{2}$ both affect the convergence properties. For simplicity, we fix a small enough $\varepsilon_{2}=0.01$, and only tune the size of $\varepsilon_{1}$. Similar results can be observed by fixing $\varepsilon_{1}$ and tuning $\varepsilon_{2}$, or tuning both $\varepsilon_{1}$ and $\varepsilon_{2}$. As shown in Figure 2, when the value of $\varepsilon_{1}$ increases from 0.01 to 0.3 , we observe faster convergence. However, when we further increase $\varepsilon_{1}$ beyond 0.4 , an oscillatory behavior takes place.

\section{B. Voltage regulation}

Consider setting $\varepsilon_{1}=\varepsilon_{2}=0.01$ and sufficient iterations. Figure 3 illustrates a snapshot of voltage magnitudes of all nodes at noon in three scenarios: (a) no voltage regulation
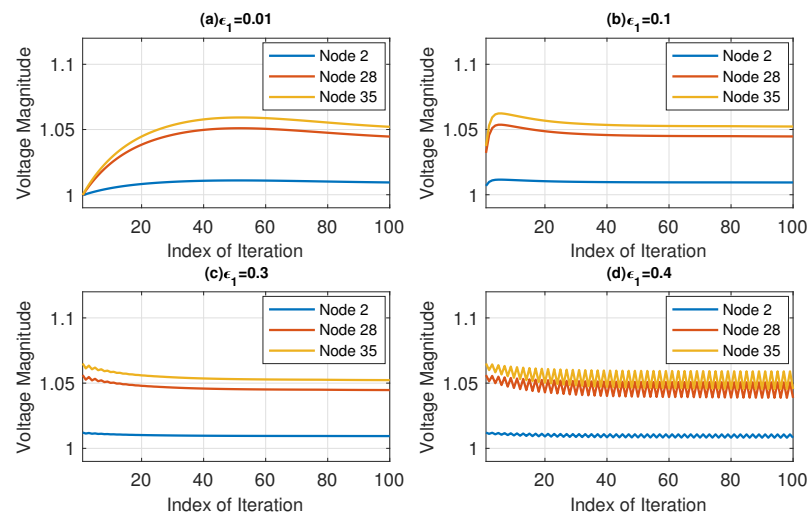

Fig. 2: Convergence of the distributed algorithm with increasing stepsize $\varepsilon_{1}$ and fixed stepsize $\varepsilon_{2}$.

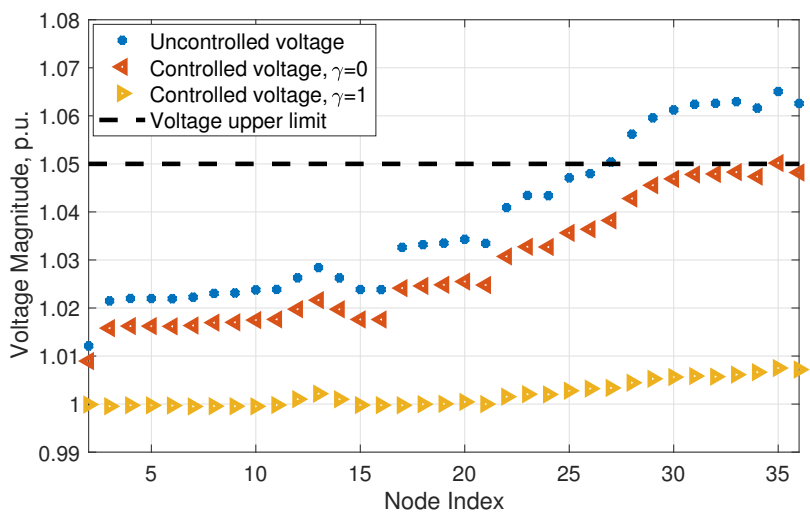

Fig. 3: Controlled/Uncontrolled voltages at all buses at noon.

algorithm applied (blue dots), (b) iterative algorithm implemented with $\gamma=0$ (red triangles), and (c) iterative algorithm implemented with $\gamma=1$ (yellow triangles).

Compared with the uncontrolled scenario (a) where voltage values would exceed the acceptable voltage limit (black dashed line) due to reverse power flows, scenarios (b) and (c) achieve voltage values that are within voltage limits. Furthermore, voltage magnitudes achieved by (c) are closer to the nominal value than those by (b), because (c) additionally penalizes voltage deviation from a nominal value of 1 p.u.

\section{CONCLUSION}

We have developed an iterative distributed algorithm that allows the distribution network operator and the endcustomers to coordinate with private information preserved in order to optimize the given economic and operational objectives while concurrently ensuring that the voltage magnitudes are within the prescribed limits. Convergence to the unique optimizer of a regularized Lagrangian associated with a well-defined social welfare maximization problem was established. We have further analyzed the performance of the proposed algorithm within nonlinear power-flow model. Numerical examples were provided to support the theoretical results. 


\section{REFERENCES}

[1] S. Maharjan, Q. Zhu, Y. Zhang, S. Gjessing, and T. Basar, "Dependable demand response management in the smart grid: A Stackelberg game approach," IEEE Trans. on Smart Grid, vol. 4, no. 1, pp. 120-132, 2013.

[2] W. Tushar, J. A. Zhang, D. B. Smith, H. V. Poor, and S. Thiébaux, "Prioritizing consumers in smart grid: A game theoretic approach," IEEE Trans. on Smart Grid, vol. 5, no. 3, pp. 1429-1438, May 2014.

[3] Y. Wang, X. Lin, and M. Pedram, "A Stackelberg game-based optimization framework of the smart grid with distributed PV power generations and data centers," IEEE Trans. on Energy Conversion, vol. 29, no. 4, pp. 978-987, Dec. 2014.

[4] S. Li, W. Zhang, J. Lian, and K. Kalsi, "Market-based coordination of thermostatically controlled loads - part i: A mechanism design formulation," IEEE Trans. on Power Systems, vol. 31, no. 2, pp. 11701178, 2016.

[5] N. Li, L. Chen, and S. H. Low, "Demand response in radial distribution networks: Distributed algorithm," Proc. of IEEE Asilomar Conference on Signals, Systems and Computers (ASILOMAR), pp. 1549-1553, 2012.

[6] — - "Optimal demand response based on utility maximization in power networks," Proc. of IEEE Power and Energy Society General Meeting, pp. 1-8, 2011.

[7] N. Li, "A market mechanism for electric distribution networks," Proc. of IEEE Conference on Decision and Control (CDC), pp. 2276-2282, Dec 2015.

[8] S. Guggilam, E. Dall'Anese, Y. Chen, S. Dhople, and G. B. Giannakis, "Scalable optimization methods for distribution networks with high PV integration," IEEE Trans. on Smart Grid, 2016.

[9] S. Dhople, S. Guggilam, and Y. Chen, "Linear approximations to ac power flow in rectangular coordinates," Proc. of IEEE Annual Allerton Conference on Communication, Control, and Computing (Allerton), 2015.

[10] S. Bolognani and S. Zampieri, "On the existence and linear approximation of the power flow solution in power distribution networks," IEEE Trans. on Power Systems, 2015.

[11] S. Bolognani and F. Dörfler, "Fast power system analysis via implicit linearization of the power flow manifold," Proc. of IEEE Annual Allerton Conference on Communication, Control, and Computing (Allerton), 2015.

[12] Y. Liu, J. Bebic, B. Kroposki, J. de Bedout, and W. Ren, "Distribution system voltage performance analysis for high-penetration PV," Proc. of IEEE Energy 2030 Conference, Nov. 2008.

[13] M. Farivar, L. Chen, and S. Low, "Equilibrium and dynamics of local voltage control in distribution systems," Proc. of IEEE Conference on Decision and Control (CDC), pp. 4329-4334, 2013.

[14] M. Farivar, X. Zhou, and L. Chen, "Local voltage control in distribution systems: An incremental control algorithm," Proc. of IEEE International Conference on Smart Grid Communications (SmartGridComm), pp. 732-737, 2015.

[15] X. Zhou, M. Farivar, and L. Chen, "Pseudo-gradient based local voltage control in distribution networks," Proc. of IEEE Annual Allerton Conference on Communication, Control, and Computing (Allerton), pp. 173-180, 2015.

[16] X. Zhou and L. Chen, "An incremental local algorithm for better voltage control in distribution networks," Proc. of IEEE Conference on Decision and Control (CDC), pp. 2396-2402, 2016.

[17] H. Zhu and H. J. Liu, "Fast local voltage control under limited reactive power: Optimality and stability analysis," IEEE Trans. on Power Systems, 2015.

[18] B. Zhang, A. D. Domínguez-García, and D. Tse, "A local control approach to voltage regulation in distribution networks," Proc. of IEEE North American Power Symposium (NAPS), Sept. 2013.

[19] M. Farivar, R. Neal, C. Clarke, and S. Low, "Optimal inverter VAR control in distribution systems with high PV penetration," Proc. of IEEE Power and Energy Society General Meeting, Jul. 2012.

[20] E. Dall'Anese, S. V. Dhople, and G. B. Giannakis, "Optimal dispatch of photovoltaic inverters in residential distribution systems," IEEE Trans. on Sustainable Energy, vol. 5, no. 2, pp. 487-497, Apr. 2014.

[21] A. Bernstein, N. J. Bouman, and J.-Y. Le Boudec, "Design of resource agents with guaranteed tracking properties for realtime control of electrical grids," 2015, [Online] Available at: http://arxiv.org/abs/1511.08628.
[22] M. Farivar, L. Chen, and S. Low, "Equilibrium and dynamics of local voltage control in distribution systems," Proc. of IEEE Conference in Decision and Control (CDC), pp. 4329-4334, December 2013.

[23] E. Dall'Anese, H. Zhu, and G. B. Giannakis, "Distributed optimal power flow for smart microgrids," IEEE Trans. on Smart Grid, vol. 4, no. 3, pp. 1464-1475, Sep. 2013.

[24] L. Gan and S. H. Low, "Convex relaxations and linear approximation for optimal power flow in multiphase radial networks," Proc. of IEEE Power System Computation Conference (PSCC), August 2014.

[25] J. Koshal, A. Nedić, and U. Y. Shanbhag, "Multiuser optimization: Distributed algorithms and error analysis," SIAM J. on Optimization, vol. 21 , no. 3, pp. 1046-1081, 2011.

[26] A. Simonetto and G. Leus, "Double smoothing for time-varying distributed multiuser optimization," Proc. of IEEE Global Conference on Signal and Information Processing (GlobalSIP), pp. 852-856, 2014.

[27] D. P. Bertsekas and J. N. Tsitsiklis, Parallel and Distributed Computation: Numerical Methods. Englewood Cliffs, NJ: Prentice Hall, 1989.

[28] X. Zhou, J. Tian, L. Chen, and E. Dall'Anese, "Local voltage control in distribution networks: A game-theoretic perspective," Proc. of IEEE North American Power Symposium (NAPS), 2016.

[29] H.-D. Chiang and M. E. Baran, "On the existence and uniqueness of load flow solution for radial distribution power networks," IEEE Trans. on Circuits and Systems, vol. 37, no. 3, pp. 410-416, 1990.

[30] J. Bank and J. Hambrick, "Development of a high resolution, real time, distribution-level metering system and associated visualization modeling, and data analysis functions," National Renewable Energy Laboratory, Tech. Rep. NREL/TP-5500-56610, May 2013. 